\title{
MUSICOTERAPIA COMO FERRAMENTA TERAPÊUTICA NO SETOR DA SAÚDE: UMA REVISÃO SISTEMÁTICA
}

\author{
Marilise Fátima de OLIVEIRA ${ }^{1}$ \\ Gleidson Brandão OSELAME ${ }^{2}$ \\ Eduardo Borba NEVES ${ }^{3}$ \\ Elia Machado de OLIVEIRA ${ }^{4}$
}

${ }^{1}$ Enfermeira. Centro Universitário Campos de Andrade. Curitiba/PR.
1 Enfermeiro. Mestre em Engenharia Biomédica. Centro Universitário Campos de Andrade. Curitiba/PR.
${ }^{1}$ Fisioterapeuta. Doutor em Saúde Pública. Centro Universitário Campos de Andrade. Curitiba -PR.
${ }^{1}$ Enfermeira. Mestre em Cirurgia. Centro Universitário Campos de Andrade. Curitiba -PR.

Recebido em: 22/09/2014 - Aprovado em: 27/11/2014 - Disponibilizado em: 15/12/2014

\begin{abstract}
Resumo
Dentre as terapias complementares e integrativas mais usadas destaca-se a música como recurso terapêutico, promovendo alterações físicas, mentais e sociais, repercutindo na recuperação e resposta ao tratamento de diversas enfermidades. Neste sentido, objetivou-se identificar as especialidades do setor da saúde em que a musicoterapia vem sendo aplicada como ferramenta terapêutica e seus benefícios. Tratou-se de um estudo de revisão sistemática realizada na base de dados do Scientific Electronic Library Online (Scielo), Literatura Latina Americana em Ciência de Saúde (LILACS) e Base de Dados de Enfermagem (BDENF), utilizando-se o descritor "musicoterapia". Observou-se o uso da música nas especialidades: Ginecologia, obstetrícia e neonatologia $(n=3)$; Nefrologia $(n=1)$; Otorrinolaringologia $(n=2)$; Cardiologia $(n=3)$; Neurologia $(n=1)$; Pediatria $(n=1)$ e Oncologia e cuidados paliativos $(n=4)$. Emergiram ainda estudos que apontavam os benefícios da música $(n=4)$. Os estudos demonstraram que a musicoterapia proporciona sensações de alegria, felicidade, bem-estar, relaxamento, mudança de rotina, entretenimento, redução de sintomas, recordações positivas, companhia e sensação de passagem mais rápida do tempo. Quando aplicada em pacientes terminais e familiares, demonstrou que a utilização da música promove a comunicação e melhora o relacionamento interpessoal entre o doente e sua família. A arte da terapêutica da musicoterapia traz a possibilidade da inovação no atendimento proporcionando qualidade de vida na evolução do tratamento. Portanto, a musicoterapia pode ser uma alternativa eficaz, independente do profissional que a empregue.
\end{abstract}

Palavras-chave: Musicoterapia, Terapêutica, Humanização.

\section{INTRODUÇÃO}

No sentido de promover a saúde é fundamental uma abordagem interdisciplinar, objetivando a prevenção, tratamento e cura de agravos de saúde.
Atualmente estas ações extrapolam ambientes como consultórios, hospitais ou unidades de saúde. Destaca-se que seja qual for o ambiente, cada vez mais atividades complementares ao tratamento estão sendo inseridas (1-3). 
Dentro da área da saúde, atividades complementares são entendidas como um conjunto de cuidados e práticas como parte das atividades e técnicas convencionais (4). Atualmente, houve grandes avanços no uso de práticas integrativas e terapêuticas no tratamento para reabilitação da população, dentre elas: hidroginástica, acupuntura, Yoga, meditações, massagens e música. Práticas estas que beneficiam a saúde do individuo(4).

Dentre as terapias complementares e integrativas mais usadas destaca-se a música como recurso terapêutico. Esta visa à qualidade de vida do sujeito em situações que promove alterações físicas, mentais e sociais, repercutindo na recuperação e resposta ao tratamento de diversas enfermidades. Sabe-se que há milênios, nas culturas antigas a música era usada como recurso terapêutico para diminuir o sofrimento(5). Foi utilizada na guerra da Criméia, por Florence Nightingale, como tratamento para amenizar a dor (6).

Como forma de comunicação, a música a partir de aspectos emocionais, produz sentimentos variados, e pode trazer conforto a quem ouve, induz o indivíduo à percepção de si na sua singularidade. Tal fato relaciona-se com a anatomia do sistema auditivo que em relação com as outras partes do corpo, por meio de suas conexões e extensões influencia a circulação, digestão, respiração e nutrição (6).

A área de atuação da musicoterapia é muito ampla, podendo beneficiar desde crianças até mesmo pessoas idosas. Como recurso utilizado com as crianças hospitalizadas evidenciou-se os benefícios da música para seus familiares, equipe de saúde e em especial as crianças. Este recurso pode ser aplicado na área da saúde como uma intervenção de baixo custo, nãofarmacológica e não-invasiva, promovendo um processo de desenvolvimento que visa à saúde da criança, da família e dos trabalhadores (7).

Em um estudo realizado com recém nascidos pré termos, objetivou-se descrever as respostas fisiológicas quando submetidos à musicoterapia clássica. Realizaram-se sessões de musicoterapia durante 15 minutos duas vezes por semana, no período vespertino e matutino. Alguns dos benefícios observados foram à redução da pressão arterial sistólica e diastólica e a melhora da frequência cardíaca e respiratória e temperatura corporal (8).

Em um estudo realizado com idosos, objetivou-se descrever os efeitos da 
utilização da música como ferramenta do cuidado a pessoa idosa. Os resultados evidenciaram a pertinência do tema para uso na promoção da saúde da população idosa e alertando a necessidade de intervenção pelos profissionais da saúde (9). No entanto, não há ainda um consenso sobre o uso da música quanto aos seus benefícios e muitos setores da saúde ainda a desconhecem como ferramenta terapêutica. Desta forma, o objetivo do presente artigo foi identificar as especialidades do setor saúde em que a musicoterapia vem sendo aplicada como ferramenta terapêutica e seus benefícios aos pacientes.

\section{METODOLOGIA}

Optou-se pela revisão sistemática qualitativa, a qual utiliza métodos para sintetizar o conhecimento das pesquisas e metodologias utilizadas nos estudos primários, sejam eles qualitativos ou quantitativos, por meio de análise descritiva (10). Constitui-se em um valioso método para a melhoria da assistência, visto que potencializa a atualização dos profissionais da saúde ao sintetizar o conhecimento acerca de um tema especifico, de forma objetiva e reproduzível. Portanto, destacar evidências acerca de práticas estimula-se a aplicação de resultados de pesquisa na prática assistencial (11).

Para nortear a revisão sistemática utilizaram-se os sete passos indicado pelo Cochrane Handbook. Este se trata de um modelo para que os pesquisadores acompanhem as etapas para realizar a seleção dos artigos; formulação da pergunta que irá delinear a pesquisa; busca e seleção dos estudos; avaliação criteriosa dos estudos; coleta e síntese dos dados (12).

A revisão orientou-se a partir da seguinte pergunta: Quais as especialidades do setor saúde em que a musicoterapia vem sendo aplicada como ferramenta terapêutica $e$ quais os benefícios do método nas diferentes patologias e populações?

Os dados coletados abrangeram as produções científicas sobre musicoterapia produzida entre os anos de 2005 a 2014, indexados em banco de dados eletrônicos da Biblioteca Virtual em Saúde, utilizando-se de todas as bases de dados nacionais e internacionais disponíveis na Literatura Latina- Americana em Ciência de Saúde (LILACS), na Base de dados de Enfermagem (BDENF) e na Scientific Electronic Library Online (SciELO). A busca foi realizada por intermédio do descritor 'musicoterapia", conforme a 
classificação dos Descritores em Ciências da Saúde (DeCS, 2014).

Para a seleção dos artigos, foram estabelecidos como critérios de inclusão: artigos publicados e indexados nas bases de dados selecionadas, publicados entre 2005 a 2014; com acesso do texto na íntegra online e gratuitamente. $\mathrm{O}$ recorte histórico escolhido justifica-se pelo interesse em abranger os artigos publicados desde a criação até a atual implementação da Política Nacional de Humanização.
A partir dos critérios de inclusão, a seleção dos artigos resultou em três fases: 1) exclusão das publicações repetidas nas bases de dados; 2) leitura do título e resumo das publicações restantes, com exclusão daquelas que não atendiam aos objetivos desta revisão; 3) avaliação criteriosa dos artigos através da sua leitura na íntegra, seguida da elaboração de tabelas sinópticos com os dados coletados. A Figura 1 apresenta os passos referentes à seleção dos artigos.
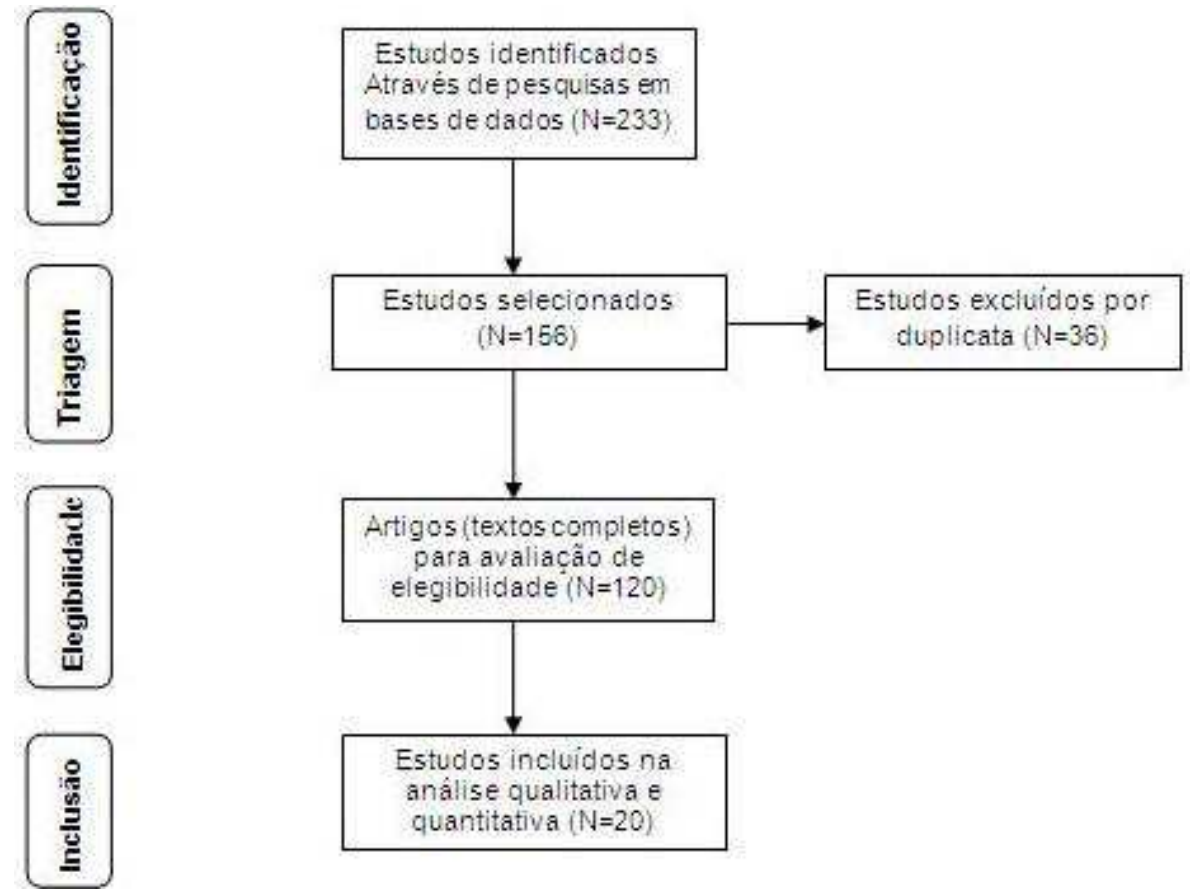

Figura 1 - Fluxograma do processo de seleção dos estudos.

Posteriormente, os dados foram seleção dos artigos; e 2) análise analisados em duas etapas: 1) processo de contemplando o seguinte aspecto: 
observação dos artigos que não utilizaram na construção ou resultados da pesquisa a palavra terapia ou terapêutica, pois na questão norteadora evidencia-se: aplicada como ferramenta terapêutica.

\section{RESULTADOS}

Com relação à musicoterapia aplicada como ferramenta terapêutica, resultaram estudos nas seguintes especialidades: Ginecologia, obstetrícia e neonatologia $(\mathrm{n}=3) ; \quad$ Nefrologia $\quad(\mathrm{n}=1)$; Otorrinolaringologia $\quad(n=2) ; \quad$ Cardiologia $(n=3)$; Neurologia $(n=1)$; Pediatria $(n=1)$ e Oncologia e cuidados paliativos $(n=4)$. Emergiram ainda estudos que apontavam os benefícios da música $(n=4)$.

Os autores possuíam como formação acadêmica a Enfermagem $(n=23)$; medicina $(n=6) ; \quad$ a odontologia $(n=5) ;$ professor universitário $\quad(n=4) ;$ fisioterapia $\quad(n=4)$; fonoaudiologia $(n=2)$. Considerando a proporção de artigos publicados por ano de publicação, observou-se um crescimento no ano de $2011(\mathrm{n}=11)$, seguido por um declínio nas publicações relacionadas ao tema no ano de $2014(n=1)$.

\section{DISCUSSÃO}

Na vida da população a música está presente tradicionalmente em vários rituais importantes. Desde melodias apropriadas para aniversário, músicas para festas cívicas, casamento, músicas de ninar, para rituais religiosos, para funerais, dentre outros. Assim, encontrada nas populações mais primitivas e nas desprovidas de tecnologias, descrita em todas as culturas do mundo (13). As especialidades que citam o uso da música como ferramenta terapêutica e seus benefícios são apresentadas a seguir:

\section{Ginecologia, obstetrícia e neonatologia}

A música foi aplicada no trabalho de parto. As gestantes foram entrevistadas após o parto e constatou-se que a música minimizou os desconfortos do parto e facilitou a adaptação do bebê nos primeiros meses de vida (13).

Outro estudo revelou que a intervenção da música na unidade de terapia intensiva neonatal (UTINEO) pode trazer benefícios para lactentes e para as mães como redução dos comportamentos de estresse, avaliados com expressões faciais de prazer (vocalização, sorriso, sucção, reflexo cócleopalpebral), ganho de peso, melhora as 
saturação de oxigênio, regulação da frequência cardíaca, respiratória e temperatura corporal (14-15).

\section{Nefrologia}

Em uma pesquisa realizada no município de Alfenas, estado de Minas Gerais, no ano de 2006 em uma clinica de hemodiálise, foram avaliados 30 sujeitos que realizavam hemodiálise na mesma sala com música ao fundo. Nestes sujeitos, mostrouse uma terapia complementar positiva proporcionando sensações de alegria, felicidade, bem-estar, relaxamento, mudança de rotina, entretenimento, ausência de sintomas, recordações positivas, companhia e o tempo passou mais rápido após a intervenção. Assim de acordo com os relatos dos sujeitos, concluíram um efeito terapêutico satisfatório (16).

\section{Otorrinolaringologia}

No estudo de Yina Quique (17) objetivaram documentar a reabilitação de pacientes com deficiência auditiva, perda de implantes cocleares e outros aparelhos auditivos. Evidenciou-se a comunicação entre a musicoterapia e a fonoaudiologia como método de terapia auditivo-oral aos pacientes. A pesquisa propiciou novos conhecimentos para intervenção com a população que utiliza implantes cocleares e outros aparelhos auditivos.

Quando utilizada em ambulatório de otorrinolaringologia, a música teve grande influencia em um caso de um menino de um ano e 10 meses, prematuro de 36 semanas, que até o seis meses não tinha emitido sons, pouca fala e andar desequilibrado. Com um ano de idade foi reavaliado pela otorrinolaringologista quanto à função auditiva e paralelamente ao tratamento médicos convencionais, participou de quatro sessões semanais de musicoterapia. Seu desenvolvimento de sociabilidade melhorou além de aprender a falar palavras novas a cada sessão, andou com mais equilíbrio, dançava e cantava cantigas de crianças, embalando chocalhos infantis com entusiasmo cooperando para seu desenvolvimento neuromotor (18).

\section{Cardiologia}

A música também demonstrou influencia no sistema cardiovascular. Evidenciou-se a melhora da variabilidade da 
frequência cardíaca em pacientes com câncer de mama pela liberação de dopamina no sistema estriatal induzida por músicas prazerosas envolvido na regulação autonômica cardíaca (19).

Noutro estudo que pesquisou a influência do sistema cardiovascular e estimulação auditiva por meio de música, indicou a melhora da variabilidade cardíaca dos pacientes (20).

O uso da música foi avaliado em um grupo de hipertensos de ambos os sexos, maiores de 50 anos e em uso contínuo de medicamentos. Participaram de sessões musicoterápicas semanais por doze semanas. Após este período, observou-se melhora significativa na qualidade de vida e controle da pressão arterial, sinalizando que essa atividade pode ter uma abordagem de atendimento multidisciplinar aos pacientes hipertensos (21).

\section{Neurologia}

Um estudo realizado por Côrte et al., (22) objetivou-se utilizar a música como atividade terapêutica para pessoas com doença de Parkinson. O estudo foi realizado com dez pacientes da Associação Brasil Parkinson (ABP). Evidenciou-se que o paciente conviveu melhor com a doença de Parkinson, minimizando assim seu sofrimento, implicando numa melhor adesão ao tratamento e beneficiando a relação como os profissionais e familiares que o cercam.

\section{Pediatria}

Em um estudo que objetivou reconhecer a eficiência da musicoterapia no controle da ansiedade durante o tratamento odontológico em pacientes com síndrome de Down, com participação voluntária de 30 crianças de 5 a 14 anos de idade, dividas em dois grupos de 15, grupo controle e grupo exposto à música. Durante a consulta odontológica, foi utilizada a escada analógica de caras como forma de avaliação, e ao comparar o nível de ansiedade e comportamento dos grupos, ficou evidente que no grupo exposto à música, $80 \%$ das crianças não apresentaram sinais de ansiedade. Já no grupo controle apenas $27 \%$ das crianças não apresentaram sinais de ansiedade. Desta forma, a musicoterapia diminuiu significativamente os níveis de ansiedade no grupo exposto a música (23). 


\section{Oncologia e cuidados paliativos}

Num estudo qualitativo de Sales et al., (24) objetivaram compreender a influência das vivências musicais na saúde física e mental de familiares de pacientes terminais. A forma metodológica escolhida foi à entrevista e observação. Os resultados demonstraram que a utilização da música contempla os preceitos filosóficos e humanitários dos cuidados paliativos, utilizado como recurso complementar para promover comunicação e melhorar o relacionamento interpessoal entre o doente e sua família.

Um estudo semelhante com acompanhantes de pacientes com câncer e tratamento antineoplásico em uma casa de apoio, constatou-se que a música proporciona aos familiares um momento essencial no enfrentamento de sua condição e percepção de suas necessidades existenciais e espirituais, ampliando as possibilidades de integração e humanização (25).

Em uma pesquisa realizada na casa de apoio da rede feminina de combate ao câncer de Maringá, no ano de 2011, as usuárias participaram em oito encontros musicais. Em seguida, foram avaliados por entrevistas individuais e constatou-se que a utilização da música proporcionou sensações de cuidado, estimulando ao tratamento e a aceitação da patologia (26).

A música também foi implantada para avaliar os efeitos no alívio da dor de pacientes oncológicos com dor crônica e identificar se ocorrem alterações nos sinais vitais. Foram avaliados dez pacientes, em que houve redução de todos em relação à intensidade da dor e estabilização dos sinais vitais. Os pacientes relataram sensação de relaxamento, alivio da dor e enxergaram na música uma forma de terapia (27).

\section{Benefícios da música}

A musicoterapia é uma forma terapêutica que utiliza a música para facilitar e promover a comunicação, aprendizagem, mobilização, inter-relação, expressão, organização, abrangendo outros alvos terapêuticos relevantes, a fim de atender as necessidades físicas, mentais, emocionais, cognitivas e sociais (17).

Em um estudo realizado por Valencia et al., (28) no México, foram comparadas três formas terapêuticas (psicossociais, a musicoterapia e as terapias múltiplas). Foram inclusos 58 pacientes que sofriam de 
esquizofrenia e que receberam a terapia por 6 meses. Foi evidenciado que a terapia psicossocial e musicoterapia tiveram um maior grau de adesão terapêutica e taxas de recaída e reinternação menores.

No estudo conduzido por Da Rocha et al., (29) buscaram compreender o funcionamento do cérebro humano utilizando a música. Utilizaram uma revisão de trabalhos internacionais publicados na última década, que relacionavam a música e neurociência. Concluíram que há benefícios da neurociência para o campo da musicoterapia como método de reabilitação.

Em outro estudo de Da Fonseca et al., (30) objetivou analisar a credibilidade e aceitação da musicoterapia aos clientes e percepção dos profissionais musicoterapeutas. A pesquisa foi desenvolvida entre 2003 e 2004, em Goiânia. Verificou-se que a maioria dos profissionais acredita na música como ferramenta terapêutica. Destacaram ainda que a música seja capaz de transmitir sensações agradáveis e atua de forma eficaz no processo de cura de algumas doenças. Ressalta-se, no entanto, a necessidade de ser divulgada com maior eficácia para a população.

\section{CONCLUSÃO}

Constatou-se diversas influências da música nas diferentes populações e patologias, bem como suas potencialidades como parte do cuidado terapêutico. As estratégias descritas contribuem para a melhoria da assistência no setor da saúde, constituindo um pilar para a humanização e colaborando na reestruturação do processo de promoção de saúde.

A música se mostrou capaz de proporcionar relaxamento, distração, bemestar, recordações agradáveis e conforto. Destaca-se negativamente o declínio ocorrido nos últimos anos de publicações acerca da temática.

São várias as áreas da saúde que usam a música como um recurso terapêutico, principalmente com crianças, pacientes oncológicos e estimulação autonômica cardíaca e mecanismo auditivo. Para este trabalho se desenvolva é fundamental ciências como a enfermagem, medicina, odontologia, fisioterapia, fonoaudiologia e outros. De forma multidisciplinar, fazendo com que o tratamento seja mais eficaz.

Portanto, o uso da música e uma terapêutica complementar valiosa, que exerce influência sobre aspectos neurocognitivos, emocionais, psíquicos e 
sociais dos pacientes, desempenhando importante papel na manutenção e melhora da qualidade de vida, além de propiciar maior interação com o meio social e familiar.

\section{REFERÊNCIAS}

1. Silva MAM, Pinheiro AKB, Souza A, Moreira ACA. Promoção da saúde em ambientes hospitalares. Rev Bras Enferm. 2011;64(3):596-9.

2. Aguiar ASC, Mariano MR, Almeida LS, Cardoso MVLML, Pagliuca LMF, Rebouças CBdA. The nurses' perceptions regarding health promotion in the Intensive Care Unit. Revista da Escola de Enfermagem da USP. 2012;46(2):428-35.

3. Verdi M, Caponi S. Reflexões sobre a promoção da saúde numa perspectiva bioética. Texto Contexto Enferm. 2005;14(1):82-8.

4. Kozasa EH. Medicina complementar e alternativa (MCA). Saúde Coletiva. 2009;6(28):38.

5. Bergold LB, Alvim NAT. A música terapêutica como uma tecnologia aplicada ao cuidado e ao ensino de enfermagem. Esc Anna Nery Rev Enferm. 2009;13(3):537-42. 6. De Carvalho Gonçalez DF, De Oliveira Nogueira AT, Puggina ACG. O uso da música na assistência de enfermagem no Brasil: uma revisão bibliográfica. Cogitare enferm. 2008;13(4):591-6.

7. Torres MCAR, Leal CMF. Reflexões de professoras supervisoras de estágios supervisionados de Música no ambiente hospitalar: desafios e aprendizagens. Revista da FUNDARTE. 2014;13(26):48-58.

8. Silva CM, Cação JMR, Silva KCS, Marques CF, Merey LSF. Respostas fisiológicas de recém-nascidos pré-termo submetidos à musicoterapia clássica. Rev Paul Pediatr. 2013;31(1):30-6.

9. Gomes L, Amaral JB. Os efeitos da utilização da música para os idosos: revisão sistemática. Revista Enfermagem Contemporânea. 2012;1(1):103-17.

10. Urra Medina E, Barría Pailaquilén RM. Systematic review and its relationship with evidence-based practice in health. Revista Latino-Americana de Enfermagem. 2010;18(4):824-31.

11. Galvão C, Sawada N, Trevizan M. Revisão sistemática. Rev Latino-am Enfermagem. 2004;12(3):549-56.

12. Higgins J, S. G. Cochrane handbook for systematic reviews of interventions Version 5.1.0; Available from: www.cochrane-handbook.org.

13. Tabarro CS, Campos L, Galli NO, Novo NF, Pereira VM. Efeito da música no trabalho de parto e no recém-nascido. Rev Esc Enferm USP. 2010;44(2):445-52.

14. Arnon S. Music therapy intervention in the neonatal intensive care unit environment. Jornal de pediatria. 2011;87(3):183-5.

15. Andriola YM, Oliveira BRG. A influência da música na recuperação do recém-nascido prematuro na UTI neonatal. Nursing (São Paulo). 2006;8(99):973-8.

16. Silva SA, Fava SMCL, Nascimento MC, Ferreira CS, Marques NR, Alves SM. Efeito terapêutico da música em portador de insuficiência renal crônica em hemodiálise. Rev enferm UERJ. 2008;16(3):382-7.

17. Yina Quique B. Métodos unisensoriales para la rehabilitación de la persona con implante coclear y métodos musicoterapéuticos como nueva herramienta de intervención. Revista de otorrinolaringología y cirugía de cabeza y cuello. 2013;73(1):94-108.

18. Picado SBR, Sousa LCA, Bugalho Filho A. Musicoterapia no ambulatório de 
otorrinolaringologia. Pediatria (São Paulo). 2010;32(1):71-4.

19. Valenti VE, Guida HL, Frizzo AC, Cardoso AC, Vanderlei LCM, Abreu LC. Auditory stimulation and cardiac autonomic regulation. Clinics. 2012;67(8):955-8.

20. Valenti VE, Guida HL, de Mello Monteiro CB, Vanderlei LCM, Ferreira LL, Ferreira $\mathrm{C}$, et al. Relationship between cardiac autonomic regulation and auditory mechanisms: importance for growth and development. Journal of Human Growth and Development. 2013;23(1):94-8.

21. Zanini CRO, Jardim PCBV, Salgado CM, Nunes MC, Urzêda FL, Carvalho MVC, et al. Music therapy effects on the quality of life and the blood pressure of hypertensive patients. Arquivos brasileiros de cardiologia. 2009;93(5):534-40.

22. Côrte B, Neto L. A musicoterapia na doença de Parkinson. Cien Saude Colet [Internet]. 2009;14(6):2295-304.

23. Gómez Scarpetta RÁ, Durán Arismendy L, Cabra Sosa LJ, Pinzón Vargas CT, Rodríguez Becerra NR. Musicoterapia para el control de ansiedad odontológica en niños con síndrome de down; Music therapy to control dental anxiety in children with Down syndrome; Musicoterapia para o controle de ansiedade odontológica em crianças com síndrome de down. Hacia promoc salud. 2012;17(2):13-24.

24. Sales CA, Silva V, Pilger C, Marcon

SS. A música na terminalidade humana: concepções dos familiares. Rev esc enferm USP. 2011;45(1):138-45.

25. Da Silva VA, MarconI SS, SalesI CA. Percepções de familiares de pessoas portadoras de câncer sobre encontros musicais durante o tratamento antineoplásico. Rev Bras Enferm. 2014;67(3):408-14.

26. Da Silva VA, Sales CA. Encontros musicais como recurso em cuidados paliativos oncológicos a usuários de casas de apoio. Revista da Escola de Enfermagem da USP. 2013;47(3):626-33.

27. Franco M, Rodrigues AB. Music therapy in relief of pain in oncology patients. Einstein (São Paulo). 2009;7(2):147-51.

28. Valencia M, Murow E, Luisa Rascón M. Comparación de tres modalidades de intervención en esquizofrenia: terapia psicosocial, musicoteapia y terapias múltiples. Revista Latinoamericana de psicología. 2006;38(3):535-49.

29. Da Rocha VC, Boggio PS. A música por uma óptica neurocientífica. Per Musi.27:132-40.

30. Da Fonseca KC, Barbosa MA, Silva DG, da Fonseca KV, Siqueira KM, de Souza MA. Credibilidade e efeitos da música como modalidade terapêutica em saúde. Revista Eletrônica de Enfermagem. 2009;8(3):398403. 